\title{
Participação de células $T$ regulatórias (Tregs) na imunopatogênese da infecção pelo vírus da imunodeficiência humana 1 (HIV-1)
}

\author{
Regulatory $T$ cells participation in the infection \\ immunopathogenesis by the Human Immunodeficiency Virus \\ (HIV-1)
}

\author{
Isabele Kazahaya Borges ${ }^{1 *}$; Juliana Elisa Lima $^{2}$; Paula de Azevedo Oliveira \\ Milanez²; Sara Santos Bernardes²; Ionice Felipe ${ }^{3}$; Ivete Conchon Costa ${ }^{4}$; Halha \\ Ostrenski Saridakis ${ }^{5}$; Maria Angelica Ehara Watanabe ${ }^{6}$
}

Resumo

\begin{abstract}
A sobrevivência de pacientes infectados pelo vírus da imunodeficiência humana (HIV-1) é relacionada à prevenção e ao tratamento eficaz de infecções oportunistas. É conhecido que os principais parâmetros para avaliar a progressão da doença causada pelo HIV-1 são contagem de células T CD4+ e carga viral do HIV-1. Células T regulatórias têm sido foco de intensas investigações dentro do sistema imunológico como também na patogênese de diversas doenças. Sabe-se que as células $\mathrm{T}$ reguladoras (Tregs) CD4+CD25+FoxP3+ atuam na modulação da ativação imune, funcionando como mediadores críticos da homeostasia imune e da auto-tolerância. Além disso, recentes estudos têm demonstrado que as células Tregs não se limitam à prevenção de auto-imunidade, mas são importantes no controle todas as formas de respostas imunes no contexto de inflamação, infecção, alergia, transplantes e imunidade tumoral. Muitos autores têm identificado as Tregs como células efetoras benéficas no contexto da AIDS, porém há discordância. Tregs podem sustentar importante função na imunopatologia da infecção pelo HIV devido a atividade supressora sobre ativação celular e função efetora. Neste contexto, esta revisão aborda os aspectos moleculares e imunológicos das Tregs no sistema HIV.
\end{abstract}

Palavras-Chaves: Tregs. HIV. CD4. AIDS.

\begin{abstract}
The survival of patients infected with human immunodeficiency virus (HIV-1) is related to the prevention and effective treatment of opportunistic infections. It is known that the main parameters to evaluate the progression of disease caused by HIV-1 is the counting of CD4 + T cells and viral load of HIV-1. Regulatory T cells has been considered the focus of intense research within the immune system as well as in the pathogenesis of several diseases. Natural regulatory T cells (Tregs) CD4+CD25+ act in the modulation of immune activation, functioning as critical mediators of immune homeostasis and self-tolerance. Furthermore, recent studies has shown that the function of Tregs cells is not limited to the prevention of autoimmunity, but is also important to control all forms of immune responses in the context of inflammation, infection, allergy, transplantation and tumor immunity. Many authors have identified Tregs as beneficial effector cells in the context of AIDS, but other researchers disagree. Tregs
\end{abstract}

\footnotetext{
1 Mestranda do Programa de Pós-Graduação em Patologia Experimental; Graduada em Farmácia pela Universidade Estadual de Londrina (2006), Habilitação em Análises Clínicas pela Universidade Estadual de Londrina (2007) e aperfeicoamento em Biologia Molecular pela Okayama University Hospital, Japão (2008).E-mail: isabele_k@yahoo.com.br

* Autor (a) para correspondência

2 Mestrandos do Programa de Pós-Graduação em Patologia Experimental.

3 Doutorado em Ciências Biológicas (Biofísica) pela UFRJ.

4 Doutorado em Microbiologia, UEL.

5 Doutorado em Doutorado Em Ciências pela UNIFESP.

6 Doutorado em Bioquímica pela USP-Ribeirão Preto, Pós-Doutorado em Imunologia pela Fundação Hemocentro de Ribeirão Preto.
} 
can exert an important role in the immunopathology of HIV infection due to the suppressor activity on cellular activation and effector function. Thus, this review discusses the molecular and immunological aspects of Tregs in the HIV system.

Key-words: Tregs. HIV. CD4. AIDS.

\section{Introdução}

A síndrome da imunodeficiência adquirida (AIDS) foi reconhecida pela primeira veznos Estados Unidos em 1980, devido ao aumento da incidência de infecções oportunistas geralmente raras (como a pneumonia causada por Pneumocystis carinii) em homens homossexuais, os quais apresentavam uma deficiência imunológica generalizada (BARRÉSINOUSSI et al., 1983). Em 1983, o vírus causador desta doença foi isolado e denominado de vírus da imunodeficiência humana (HIV) e, anos mais tarde, foram evidenciados dois tipos de HIV: HIV1 e HIV-2, com estruturas genômicas ligeiramente diferenciadas.

O HIV-1 é um vírus citopático e não oncogênico, com genoma RNA, membro da família retroviridae e subfamília Lentivirina. Para multiplicar-se, necessita da enzima transcriptase reversa (RT), a qual é responsável pela transcrição do RNA viral para uma cópia de DNA, que pode, então, integrarse ao genoma do hospedeiro (BARRE-SINOUSSI et al., 1983; GALLO et al., 1984; LEVY et al., 1984).

A história natural da infecção pelo HIV-1 pode ser dividida em três fases clínicas, segundo Rizzardi e Pantaleo (1999): 1) infecção primária pelo HIV1; 2) fase crônica assintomática e 3) AIDS. O tecido linfático serve como principal reservatório para o HIV-1, e nele ocorre replicação viral e uma progressiva deterioração anatômica e funcional, e isso prejudica a capacidade de manutenção da resposta imune específica, refletida pelo rápido aumento dos níveis de viremia e pela queda na contagem de células TCD4+. Esses fatos possibilitam acelerar a transição da fase primária para um estágio mais avançado da doença (PANTALEO et al., 1993; PANTALEO et al., 1998).

A infecção pelo HIV-1 causa desequilíbrio do sistema imunológico por diminuição ou perda da função dos linfócitos T CD4+, como também de outras células envolvidas na apresentação e processamento de partículas virais.

A conseqüência da deterioração progressiva que ocorre com a maioria dos pacientes infectados pelo HIV-1 é o desenvolvimento de doença clinicamente aparente ou da aids. O último estágio da infecção é caracterizado pelo aparecimento ou reativação de infecções causadas por inúmeros agentes infecciosos (MARQUES; MANSUR, 2002).

A história natural e o processo patogênico da infecção pelo HIV-1 são complexos e dependem de vários fatores relacionados a interação do vírus com o hospdeiro (PANTALEO et al., 1993).

A infecção pelo HIV-1 é caracterizada por deficiência do sistema imunológico, expressa não somente pelo reduzido número de células CD4+, mas também pelo distúrbio das células $\mathrm{T}$ remescentes. Vários eventos têm demonstrado importância como a ativação imune crônica, a perda de células $\mathrm{CD} 4+$ e a conseqüente incapacidade das células remanescentes responderem a algum estímulo (TERZIEVA et al., 2009). Estes eventos são acompanhados por mudanças significativas no perfil de citocinas, numa fase precoce da infecção, e tornam-se mais significativas com a progressão da doença (TERZIEVA et al., 2009).

Células T CD4+ vírus específicas são críticas na imunidade antiviral (CORREA et al., 2007). O valor absoluto de células T CD4+ é um importante marcador para a progressão da infecção pelo HIV, como também para a restituição da resposta imune. Embora tenha sido descrito que a resposta de células $\mathrm{T}$ a antígenos apresenta-se reduzida em indivíduos infectados pelo HIV, permanece obscuro se esse fato se deve a uma diminuição nos números de células $\mathrm{T}$ ou a defeitos qualitativos nos linfócitos 
remanescentes (KNOX et al., 2006). Entretanto há muitas evidências de que a linfopenia não é o único mecanismo responsável pela disfunção imunológica, baixos níveis de células $\mathrm{T}$ CD4+ podem estar relacionados com a recuperação imunológica deficitária após o início da terapia anti-retroviral. $\mathrm{O}$ aumento nos números de linfócitos T CD4+ durante a terapia anti-retroviral não é preditivo de êxito na restituição das respostas imunes a antígenos (D’AMICO et al., 2005).

Recentemente, tem sido evidenciado que uma subpopulação de células $\mathrm{T}$, denominada células $\mathrm{T}$ regulatórias (Tregs), apresenta envolvimento com tolerância a antígenos self. O Foxp3 (Forkhead box p3) está expresso nas células Tregs CD4+CD25+e tem sido considerado um dos principais mediadores da supressão de tolerância periférica.

As Tregs foram descritas pela primeira vez em 1995 por Sakaguchi e colaboradores, como uma célula reguladora da resposta imune e da ativação celular. Treg CD4+CD25+ foram reconhecidas como supressoras das respostas de células CD4 e CD8 antígeno específicas e também como controladoras da ativação imune inapropriada ou exacerbada induzida por patógenos (BELKAID; ROUSE, 2005; RAGHAVAN; HOLMGREN, 2005).

As Tregs desempenham papel fundamental na limitação da imunopatogênese das infecções virais crônicas de estimulação imune persistente (SAKAGUCHI, 2003). Assim, talvez seu papel na resposta imune à infecção por HIV possa ser muito relevante, embora haja dados conflitantes sobre o potencial papel das células $\mathrm{T}$ regulatórias na imunopatogênese da doença causada pelo HIV. Alguns autores argumentaram que estas células reguladoras poderiam prevenir ou suprimir uma resposta imunológica eficaz (OSWALD-RICHTER et al, 2004), enquanto outros têm relatado uma associação positiva entre aumento de células Tregs, a diminuição da viremia e o aumento da contagem de células T CD4+ (KINTER et al., 2004).

\section{Células T regulatórias Foxp3+ (Tregs)}

Atualmente, as Tregs estão sendo consideradas o mais importante subtipo de células regulatórias, representando acima de 5\% dos linfócitos T CD4 do sangue periférico (WING et al., 2002). Como marcadores dessas células, pode-se citar, além da expressão constitutiva do fator de transcrição Foxp3, o receptor da família TNF induzido por glucocorticóide (GITR), o antígeno 4 associado ao linfócito T citotóxico (CTLA-4) e elevado nível da cadeia alfa do receptor de IL-2 (CD25) (CRUVINEL et al., 2008). Tregs CD4+CD25+Foxp3+ estão envolvidas na tolerância imune, autoimunidade, inflamação, transplantes, câncer e infecção pelo HIV (FONTENOT; GAVIN; RUDENSKY, 2003). Muitos autores consideram a proteína Foxp3 como o constituinte mais importante das Tregs (FONTENOT; GAVIN; RUDENSKY, 2003; HOLMES et al, 2008; FEUERER et al., 2009), já que todas Treg com atividade supressora expressam esse fator de transcrição. Esse fator, embora necessário, não é o suficiente para a geração dessas células in vitro. A proteína GITR também é considerada um marcador importante (ANDERSON et al., 2005) e, segundo Van Olffen et al. (2009), está relacionado com a proliferação das células $\mathrm{T}$, a fim de proporcionar um equilíbrio na população das células TCD4+.

Têm sido descritas duas origens para as células Foxp3+: no timo, onde estas células são produzidas passando por seleção positiva, e na periferia, onde vários fatores induzem a expressão do Foxp3 (FEUERER et al., 2009). O mecanismo de conversão CD25-CD4+ em CD25+CD4+ na periferia, in vitro, envolve principalmente o TGF-b, sendo também demonstrado que a vitamina $\mathrm{D}$, a prostaglandina E2 e a enzima indoleamina-2,3-dioxigenase (IDO) também induzem a expressão de Foxp3 e sua função supressora (GROTH; LANDAY, 2008). Nos últimos anos, têm sido publicados muitos estudos relacionados a conversão de Treg na periferia pela IDO (CURTI et al., 2007), e destacando seu papel junto ao CTLA-4. Essa proteína de superfície da 
Treg induz a produção de IDO pelas células dendritícas (MUNN et al., 2004; BOASSO et al., 2005), depletando o aminoácido essencial triptofano e liberando produtos chamados kinureninas, que são capazes de inilibir a ativação das células $T$ juntamente com outros estímulos (FALLARINO et al., 2003; BOEHMER, 2005). Estudos têm definido o TGF- $\beta$ como regulador do desenvolvimento das Tregs originadas no timo, bem como fator essencial na homeostasia das células T na periferia, tolerância para antígenos self e diferenciação das células durante a resposta imune (LI; FLAVELL, 2008).

Estudos in vitro têm demonstrado que as Tregs, quando estimuladas, são capazes de produzir pequenas quantidades de IL-10 e TGF- $\beta$, porém nenhuma dessas citocinas parece ser necessária para a sua função efetora. Essa função é mediada através de um mecanismos dependente de contato celular e independente de citocinas supressoras, sendo os dados sobre esses mecanismos ainda conflitantes. Alguns autores verificaram que culturas de células T CD4+CD25+ levam à redução de moléculas coestimulatórias CD80 e CD86 em células dendríticas e linfócitos B (SEMPERE; SORIANO; BENITO, 2007). Outros sugerem que o contato direto célula T-célula $\mathrm{T}$ é independente de células apresentadoras de antígenos (APCs), e se dá através de moléculas de superfície, como o GITR (RONCHETTI et al., 2004).

Embora as Tregs apresentem o receptor de alta afinidade para IL-2 (CD25) expresso em altas concentrações em sua superfície, o papel dessa citocina na função dessas células é ainda controverso. Alguns autores relatam que essa interleucina não possui nenhum efeito imunossupressor in vitro (BOEHMER, 2005), apesar de ser necessária no meio de cultivo para a produção das Tregs (MALOY; POWERI, 2005). Estudos clínicos recentes envolvendo a administração de IL-2 relatam um aumento do número de Tregs e também melhor prognóstico clínico, devido a esse aumento. Esse fato indica que a viabilidade e funcionalidade dessas células é suportada pela IL-2 (GROTH;
LANDAY, 2008; TERZIEVA et al., 2009).

O Foxp3 tem sido proposto como um marcador específico adicional para Tregs dentro da população de linfócitos T CD4+CD25+. Foxp3 é um membro da família de fatores de transcrição forkhead/winged que atuam através do fator nuclear de linfócitos $T$ ativados (NFAT) e controlam genes considerados primordiais no desenvolvimento das Tregs (ZHENG et al., 2007; MARSON et al., 2007). O Foxp3 parece ser um marcador universal de expressão de linfócitos $\mathrm{T}$, incluindo subtipos CD4+ e CD8+, e como mencionado acima, a expressão deste fator de transcrição pode ser necessária, mas não suficiente para desenvolvimento das Tregs.

A indução da expressão de IL-2 durante a ativação das células $\mathrm{T}$ é altamente dependente da ativação de de três fatores de ativação transcricional (NFAT, AP1 e NF-kB) que se ligam no promotor da IL-2 e ativam a expressão deste gene (HOLMES et al., 2008). O mecanismo de ação mais estudado do Foxp3 é a inibição do NFAT, AP1 e NF-kB nas células que o expressam, reduzindo ou inibindo a produção de IL-2 (CARSON et al., 2006), fazendo com que essas células usem IL-2 exógena para seu crescimento e diferenciação, e, conseqüentemente, suprimam a ativação de células produtoras dessa interleucina, como as células T efetoras (MALOY; POWERI, 2005; CRUVINEL et al., 2008). É relatado que o Foxp3 pode funcionar como um repressor da transcrição durante a ativação das células $\mathrm{T}$ através da inibição de NFAT e NF-kB, e reprime a produção de IL-2, IL-4, e IFN-g (BETTELLI; DASTRANGE; OUKKA, 2005). Lee, Gao e Fang (2008) verificaram que Foxp3 interage com c-Jun, um membro transcricional do AP-1, e inibe a ligação ao DNA, por alterar a sua localização subnuclear, e induzir a anergia nas células T. Esse fator de transcrição pode também formar um complexo com NFAT, para induzir a expressão de marcadores das Tregs como CTLA-4 e CD25, e conferir a função supressora (WU et al., 2006). 


\section{Tregs na imunopatogênese do HIV}

Tregs CD4+CD25+ desempenham função importante na limitação da imunopatologia de infecção crônica viral durante estimulação persistente. Os dados relacionados a função das Tregs na imunopatogênese da infecção pelo HIV têm sido conflitantes. Alguns autores têm argumentado que estas células previnem ou suprimem a resposta imune efetiva (LIM et al., 2007), enquanto outros têm reportado uma associação positiva entre elevada concentração de Tregs, baixa viremia e contagem elevada de células CD4+ (BAKER et al., 2007).

Estudos de Card et al. (2009) verificaram que indivíduos resistentes ao HIV apresentaram freqüência reduzida de células $\mathrm{T}$ que expressam CD69. Foi também encontrada freqüência elevada de Tregs em pacientes HIV positivos quando comparado com indivíduos saudáveis.

Tregs podem suprimir a imunidade mediada por células contra o HIV-1. Tem sido demonstrado que depleção de Tregs em pacientes infectados por HIV aumenta resposta anti-HIV de células T (EGGENA et al., 2005) e que o número de células T Foxp3+ foi significantemente aumentado em tecidos linfóides de pacientes infectados (NILSSON et al., 2006).

Bi et al. (2009) verificaram elevada freqüência e proliferação de Tregs CD4+ Foxp3+ em pacientes infectados pelo HIV, com contagem diminuída de células $\mathrm{CD} 4+$. Os autores sugeriram que Tregs ativadas no sistema HIV expressam de alguma forma o receptor de quimiocina CCR7, e favorecem, desta forma, a migração destas para os órgãos linfóides secundários.

Uma proteína do envelope do HIV, a gp120, parece estar envolvida na ativação das Tregs. Nilsson et al. (2006) analisaram o nível e função de Tregs nos tecidos linfóide de pacientes infectados pelo HIV com doença progressiva ou não. Foi encontrado aumento do número de Tregs (Foxp3+) em indivíduos HIV com doença progressiva, o qual não foi relatado com ativação do sistema imune. Este aumento de Tregs diminuiu a imunidade celular. Experimentos realizados in vitro com células Tregs CD4 expostas ao HIV inativado demonstraram que a interação CD4-gp120 promove sobrevivência destas células por inibição da apoptose, concluindo que a expansão seletiva destas células Tregs são direcionadas pelo HIV. Becker et al. (2009) observaram que a interação gp120-CD4 é usado como uma poderosa ferramenta para ativação funcional das células Tregs in vitro e in vivo.

Foi verificada diminuição de células Tregs em pacientes com infecção crônica pelo HIV. Esta observação sugere que as Tregs, como as células $\mathrm{T}$ convencionais, são progressivamente perdidas durante a infecção pelo HIV. Células de indivíduos infectados que mostram células Treg especificas para HIV in vitro tem viremia significantemente menor e elevada contagem de células CD4 e CD8 quando comparados com indivíduos com atividade Treg não detectável (KINTER et al., 2004). No entanto, a observação de que a expressão de marcadores de Tregs naturais, como Foxp3, CD25 e CTLA4, está aumentada nos tecidos linfóides de pacientes infectados pelo HIV e macacos infectados pelo SIV (simian immunodeficiency virus) sugere que o acúmulo de células Tregs em tecidos infectados pode ocorrer devido à diminuição da freqüência de Tregs naturais no sangue (ANDERSSON et al., 2005).

$\mathrm{O}$ aumento da contagem e frequência de células Tregs de mucosa parece ser um fator característico da infecção pelo HIV na ausência de tratamento, e sugere uma significante contribuição de Tregs na patogênese da doença. Essa função pode ser atenuada por hiperativação imune induzida pelo HIV, enquanto há supressão da resposta imune para HIV e patógenos da mucosa (EPPLE et al., 2006). Esse acúmulo, no entanto, pode estar associado com aumento do nível de infecção nestes tecidos. Tem sido demonstrado que células T CD4+CD25+ de culturas de células linfóides ou periféricas de pacientes infectados pelo HIV apresentam supressão da resposta imune HIV-específica (KINTER et al., 2007). Esses trabalhos sugerem que células Tregs 
naturais, podem contribuir para a replicação não controlada do vírus por supressão específica da imunidade. Ji e Cloyd (2009) propuseram um mecanismo no qual a ligação do CD4 com o HIV nas células Tregs aumenta a função imunossupressiva e induz a migração destas células da periferia aos tecido.

\section{Considerações finais}

Desde os primeiros anos da epidemia da AIDS, a contagem das células T CD4+ vem sendo utilizada como um parâmetro laboratorial preditivo do prognóstico da doença causada pelo HIV e também como um indicador do potencial de risco para as principais infecções oportunistas, principalmente em pacientes com doença sintomática. A população de células T CD4+ diminui à medida que a infecção pelo HIV progride, diminuindo a capacidade do organismo em responder contra vários microrganismos. Embora muitos pesquisadores discutam que a supressão de células efetoras específicas para o HIV não seja benéfica ao hospedeiro, muitos grupos têm verificado que as células Tregs protagonizam a limitação da ativação imune crônica associada à infecção pelo HIV, e, portanto, seriam benéficos ao hospedeiro. Até o momento, não há convicção nas pesquisas realizadas, se há vantagem do efeito no controle da ativação do sistema imunológico ou se este efeito das Tregs pode ser prejudicial causado pela supressão da resposta antiviral. Desta forma, a questão sobre as células Tregs na imunopatogênese da infecção pelo HIV permanece ainda a ser elucidada.

\section{Agradecimentos}

Programa de Pós-Graduação em Patologia Experimental, PROPPG-UEL e CAPES.

\section{Referências}

ANDERSSON, J.; BOASSO, A.; NILSSON, J.; ZHANG, R.; SHIRE, N. J.; LINDBACK, S.;.SHEARER, G. M.; CHOUGNET, C. A. Cutting edge: the prevalence of regulatory $\mathrm{T}$ cells in lymphoid tissue is correlated with viral load in HIV infected patients. The Journal of Immunology, Baltimore, v. 174, p. 3143-3147, 2005.

BAKER, C. A. R.; CLARK, R.; VENTURA, F.; JONES, N. G.; GUZMAN, D.; BANGSBERG, D. R.; CAO, H. Peripheral CD4 loss of regulatory $\mathrm{T}$ cells is associated with persistent viraemia in chronic HIV infection. Clinical and Experimental Immunology, Oxford, v. 174, n. 3, p. 533-539, 2007.

BARRE-SINOUSSI，F.; CHERMANN，J. C.; REY, F.; NUGEYRE, M. T.; CHAMARET, S.; GRUEST, J.; DAUGUET, C.;AXLER-BLIN, C.; VEZINET-BRUN, F.; ROUZIOUX, C.; ROZENBAUM, W.; MONTAGNIER, L. Isolation of a T-lymphotropic retrovirus from a patient at risk for acquired immune deficiency syndrome (AIDS). Science, Washington, v. 20, n. 220, p. 868-871, 1983.

BECKER, C.; TAUBE, C.; BOPP, T.; BECKER, C.; MICHEL, K.; KUBACH, J.; REUTER, S.; DEHZAD, N.; NEURATH, M. F.; REIFENBERG, K.; SCHNEIDER F. J.; SCHMITT, E.; JONULEIT, H. Protection from graftversus-host disease by HIV-1 envelope protein gp120mediated activation of human $\mathrm{CD} 4+\mathrm{CD} 25+$ regulatory $\mathrm{T}$ cells. Blood, New York, v. 114, n. 6, p. 1263-1269, 2009.

BELKAID, Y.; ROUSE, B.T. Natural regulatory T cells in infectious disease. Nature Immunology, New York, v. 6, n. 4, p. 353-360, 2005.

BETTELLI, E.; DASTRANGE, M.; OUKKA, M. Foxp3 interacts with nuclear factor of activated $\mathrm{T}$ cells and NFkappa B to repress cytokine gene expression and effector functions of T helper cells. Proceedings of the National Academy of Sciences of United States of America, Washington, v. 102, n. 14, p. 5138-5143, 2005.

BI, X.; SUZUKI, Y.; GATANAGA, H.; OKA, S. High frequency and proliferation of CD4+ FOXP3+ Treg in HIV-1-infected patients with low CD4 counts. European Journal of Immunology, Weinheim, v. 39, n. 1, p. 301309, 2009.

BOASSO, A.; HERBEUVAL, J. P.; HARDY, A. W.; WINKLER, C.; SHEARER, G. M. Regulation of indoleamine-2,3-dioxygenase and tryptophanyl- tRNAsynthetase by CTLA-4-Fc in human CD4R T cells. Blood, New York, v. 105, n. 5, p. 1574-1581, 2005.

BOEHMER, V. H. Mechanisms of suppression by suppression T cells. Nature Immunology, New York, v. 6, n. 4, p. 338-344, 2005. 
CARD, C. M.; MCLAREN, P. J.; WACHIHI, C.; KIMANI, J.; PLUMMER, F. A.; FOWKE, K. R. Decreased immune activation in resistance to HIV-1 infection is associated with an elevated frequency of CD4(+)CD25(+)FOXP3(+) regulatory T cells. Journal of Infectious Diseases, New York, v. 199, n. 9, p. 13181322, 2009.

CARSON, B. D.; LOPES, J. E.; SOPER, D. M.; ZIEGLER, S. F. Insights into transcriptional regulation by FOXP3. Bioscience, Washington v. 1, n. 11, p. 16071619, 2006.

CORREA, R.; HARARI, A.; VALLELIAN, F.; RESINO, S.; MUNOZ-FERNANDEZ, M. A.; PANTALEO, G. Functional patterns of HIV-1-specific CD4 T-cell responses in children are influenced by the extent of virus suppression and exposure. AIDS, London v, 21, n. 1, p. 23-30, 2007.

CRUVINEL, W. M.; MESQUITA JUNIOR.; D.; ARAÚJO, J. A. P.; SALMAZI, K. C.; KÁlLAS, E. P.; ANDRADE, L. E. C. Células T Regulatórias Naturais (TREGS) em Doenças Reumáticas Natural Regulatory $\mathrm{T}$ cells in Rheumatic Diseases. Revista Brasileira de Reumatologia, São Paulo, v. 48, n. 6, p. 342-355, 2008.

CURTI, A.; PANDOLFI, S.; VALZASINA, B.; ALUIGI, M.; ISIDORI, A.; FERRI, E.; SALVESTRINI, V.; BONANNO, G.; RUTELLA, S.; DURELLI, I.; HORENSTEIN, A.L.; FIORE, F.; MASSAIA, M.; COLOMBO, M.P.; BACCARANI, M.; LEMOLI, R. M. Modulation of tryptophan catabolism by human leukemic cells results in the conversion of CD25S into CD25R T regulatory cells. Blood, New York, v. 109, p. 2871-2877, 2007.

D’AMICO, R.; YANG, Y.; MILDVAN, D.; EVANS, S. R.; SCHNIZLEIN-BICK, C. T.; HAFNER, R.; WEBB, N.; BASAR, M.; ZACKIN, R.; JACOBSON, M. A. Lower CD4+ T lymphocyte nadirs may indicate limited immune reconstitution in HIV-1 infected individuals on potent antiretroviral therapy: analysis of immunophenotypic marker results of AACTG 5067. Journal of Clinical Immunology, v. 25, n. 2, p. 106-115, 2005.

EGGENA, M.P; BARUGAHARE, B.; JONES, N.; OKELLO, M.; MUTALYA, S.; KITYO, C.; MUGYENYI, P.; CAO, H. Depletion of regulatory T cells in HIV infectionis associated with immune activation. The Journal of Immunology, Baltimore, v. 174, n.7, p. 4407-414, 2005.

EPPLE, H. J.; LODDENKEMPER, C.; KUNKEL, D.; TRÖGER, H.; MAUL, J.; MOOS, V.; BERG, E.; ULLRICH, R.; SCHULZKE, J. D.; STEIN, H.; DUCHMANN, R.; ZEITZ, M.; SCHNEIDER, T. Mucosal but not peripheral FOXP3+ regulatory $\mathrm{T}$ cells are highly increased in untreated HIV infection and normalize after suppressive HAART. Blood, New York, v. 108, n. 9, p. 3072-3078, 2006.

FALLARINO, F.; GROHMANN, U.; HWANG, K. W.; ORABONA, C.; VACCA, C.; BIANCHI, R.; BELLADONNA, M. L.; FIORETTI, M. C.; ALEGRE, M. L.; PUCETTI, P. Modulation of tryptophan catabolism by regulatory T cells. Nature Immunology, New York, v. 4, p. 1206-1212, 2003.

FEUERER, M.; HILL, J. A.; MATHIS, D.; BENOIST, C. Foxp3+ regulatory $\mathrm{T}$ cells: differentiation, specification, subphenotypes. Nature Immunology, New York, v. 10, n. 7, p. 689-695, 2009.

FONTENOT, J. D.; GAVIN, M. A.; RUDENSKY, A. Y. FoxP3 programs the development and function of CD4+CD25 regulatory T cells. Nature Immunology, New York, v. 4, n. 4, p. 304-306, 2003.

GALLO, R. C.; SALAHUDDIN, S. Z.; POPOVIC, M.; SHEARER, G. M.; KAPLAN, M.; HAYNES, B. F.; PALKER, T. J.; REDFIELD, R.; OLESKE, J.; SAFAI, B. Frequent detection and isolation of cytopathic retroviruses (HTLV-III) from patients with AIDS and at risk for AIDS. Science, Washington, v. 224, n. 4648, p. 500-503, 1984.

GROTH, B. F, LANDAY, A. L. Regulatory T cells in HIV infection: pathogenic or protective participants in the immune response? AIDS, London, v. 22, n. 6, p. 671-683, 2008.

HOLMES, D.; JIANG, Q.; ZHANG, L; SU, L. Foxp3 and Treg cells in HIV-1 infection and immuno-pathogenesis. Immunologic Research, New York, v. 41, n. 3, p. 248266, 2008.

JI, J.; CLOYD, M. W. HIV-1 binding to CD4 on $\mathrm{CD} 4+\mathrm{D} 25+$ regulatory $\mathrm{T}$ cells enhances their suppressive function and induces them to home to, and accumulate in, peripheral and mucosal lymphoid tissues: an additional mechanism of immunosuppression. International Immunology, Oxford, v. 21, n. 3, p. 283-294, 2009.

KINTER, A.; MCNALLY, J.; RIGGIN, L.; JACKSON, R.; ROBY, G.; FAUCI, A. S. Suppression of HIVspecific $\mathrm{T}$ cell activity by lymph node CD25_regulatory $\mathrm{T}$ cells from HIV-infected individuals. Proceedings of the National Academy of Sciences of United States of America, Washington, v. 104, n. 9, p. 3390-3395, 2007.

KINTER, A. L.; HENNESSEY, M.; BELL, A.; KERN, S.; LIN, Y.; DAUCHER, M.; PLANTA, M.; MCGLAUGHLIN, M.; JACKSON, R.; ZIEGLER, S. F.; FAUCI, A. S. CD25(+)CD4(+) regulatory T cells from the peripheral blood of asymptomatic HIV-infected 
individuals regulate CD4(+) and CD8(+) HIV-specific T cell immune responses in vitro and are associated with favorable clinical markers of disease status. Journal of Experimental Medicine, New York, v. 200, n. 32, p. 331343, 2004.

KNOX, K. S.; DAY, R. B.; KOHLI, L. M.; HAGE, C. A.; TWIGG, H. L. III. Functional impairment of CD4 T cells despite normalization of T cell number in HIV. Cellular Immunology, New York, v. 242, n. 1, p. 46-51, 2006

LEE, S. M.; GAO, B.; FANG, D. FoxP3 maintains Tregs unresponsiveness by selectively inhibiting the promoter DNA-binding activity of AP-1. Blood, New York, v. 111, n. 7, p. 3599-3606, 2008.

LEVY, J. A.; HOFFMAN, A. D.; KRAMER, S. M.; LANDIS, J. A.; SHIMABUKURO, J. M.; OSHIRO, L. S. Isolation of lymphocytopathic retroviruses from San Francisco patients with AIDS. Science, Washington, v. 225 , n. 4664 , p. 840-842, 1984.

LI, M. O.; FLAVELL, R. A. TGF-beta: a master of all T cell trades. Cellular Immunology, New York, v. 134, n. 3, p. 392-404, 2008.

LIM, H.W.; BROXMEYER, H.E.; CHANG, H.; KIM, C.H. Regulation of trafficking receptor expression in human forkhead box P3+ Regulatory T Cells. The Journal of Immunology, Baltimore, v.177,n.2, p.840-51, 2006.

MALOY, K. J.; POWERI, F. Fueling regulation: IL-2 keeps T regs fit. Nature Immunology, New York, v. 6, n. 11, p. 1071-1072, 2005.

MARQUES, A.; MASUR, H. Manifestações Clinicas In: VERONESI, R.; FOCACCIA, R. Tratado de Infectologia. 2. ed. São Paulo: Atheneu, 2002. p. 99-101.

MARSON, A.; KRETSCHMER, K.; FRAMPTON, G. M.; JACOBSEN, E. S.; POLANSKY, J. K.; MACISAAC, K. D.; LEVINE, S. S.; FRAENKEL, E.; VONBOEHMER, H.; YOUNG, R. A. Foxp3 occupancy and regulation of key target genes during T-cell stimulation. Nature Immunology, New York, v. 445, n. 7130, p. 931935, 2007

MUNN, D.H; SHARMA, M.D; MELLOR, A.L. Ligation of B7-1/B7-2 by Human CD4R T cells triggers indoleamine-2,3-dioxygenase activity in dendritic cells. The Journal of Immunology, Baltimore, v.172, n.7, p.4100-110, 2004.

NILSSON, J.; BOASSO, A.; VELILLA, P. A.; ZHANG, R.; VACCARI, M.; FRANCHINI, G.; SHEARER, G. M.; ANDERSSON, J.; CHOUGNET, C. HIV-1 driven regulatory $\mathrm{T}$ cell accumulation in lymphoid tissues is associated with disease progression in HIV/AIDS. Blood, New York, v. 108, n. 12, p. 3808-3817, 2006.
OSWALD-RICHTER, K.; GRILL, S. M.; SHARIAT, N.; LEELAWONG, M.; SUNDRUD, M. S.; HAAS, D. W.; UNUTMAZ, D. HIV infection of naturally occurring and genetically reprogrammed human regulatory T-cells. Plos Biology, San Francisco, v. 2, n. 7, 2004. Disponível em: $\quad<$ http://www.ncbi.nlm.nih.gov/pmc/articles/ PMC449855/>. Acesso em: 3 ago. 2010.

PANTALEO, G.; COHEN, O. J.; SCHACKER, T.; VACCAREZZA, M.; GRAZIOSI, C.; RIZZARDI, G. P.; KAHN, J.; FOX, C. H.; SCHNITTMAN, S. M.; SCHWARTZ, D. H.; COREY, L.; FAUCI, A. S. Evolutionary pattern of human immunodeficiency virus (HIV) replication and distribution in lymph nodes following primary infection: implications for antiviral therapy. Nature Medicine, New York, v. 4, n. 3, p. 341345, 1998.

PANTALEO, G.; GRAZIOSI, C.; DEMAREST, J. F.; BUTINI, L.; MONTRONI, M.; FOX, C. H.; ORENSTEIN, J. M.; KOTLER, D. P.; FAUCI, A. S. HIV infection is active and progressive in lymphoid tissue during the clinically latent stage of disease. Nature Immunology, New York, v. 362, n. 6418, p. 355-358, 1993.

RAGHAVAN, S.; HOLMGREN, J. CD4RCD25R suppressor $\mathrm{T}$ cells regulate pathogen induced inflammation and disease. FEMS Immunology and Medical Microbiology, Amsterdam v. 44, p. 121-127, 2005.

RIZZARDI, G. P.; PANTALEO, G. The immunophatogenesis of HIV-1 infection. In: POLSKY, B. W.; CLUMECK, N. HIV and AIDS. London: MosbyWolfe Medical Communications, 1999. p. 6.1-6.12.

RONCHETTI, S.; ZOLLO, O.; BRUSCOLI, S.; AGOSTINI, M.; BIANCHINI, R.; NOCENTINI, G., AYROLDI, E.; RICCARDI, C. GITR, a member of the TNF receptor superfamily, is co-stimulatory to mouse $\mathrm{T}$ lymphocyte subpopuations. European Journal of Immunology, Weinheim, v. 34, p. 613-622, 2004.

SAKAGUCHI, S. Regulatory $\mathrm{T}$ cells: mediating compromises between host and parasite. Nature Immunology, New York, v. 4, n. 1, p. 10-11, 2003.

SEMPERE, J. M.; SORIANO, V.; BENITO, J. M. T. Regulatory Cells and HIV Infection. AIDS, London, v. 9, n. 1, p. 54-60, 2007.

TERZIEVA, V.; POPOVA, D.; KICHEVA, M.; TODOROVA, Y.; MARKOVA, R.; MARTINOVA, F.; ELENKOV, I.; YANKOVA, M. Correlation between the degree of immune activation, production of IL-2 and FOXP3 expression in CD4+CD25+ T regulatory cells in HIV-1 infected persons under HAART. International 
Immunopharmacology, Amsterdam, v. 9, n. 7/8, p. 831836, 2009.

VAN OLFFEN, R. W.; KONING, N.; VAN GISBERGEN, K. P.; WENSVEEN, F. M.; HOEK, R. M.; BOON, L.; HAMANN, J.; VAN LIER, R. A.; NOLTE, M. $A$. GITR triggering induces expansion of both effector and regulatory CD4+ $\mathrm{T}$ cells in vivo. The Journal of Immunology, Baltimore, v. 182, n. 12, p. 7490-7500, 2009.

WING, K.; EKMARK, A.; KARLSSON, H.; RUDIN, A.; PAYER, E. S. Characterization of human CD25+ CD4+ $\mathrm{T}$ cells in thymus, cord and adult blood. Immunology, Oxford, v. 106, n. 2, p. 190-199, 2002.

WU, Y.; BORDE, M.; HEISSMEYER, V.; FEUERER, M.; LAPAN, A. D.; STROUD, J. C.; BATES, D.L.; GUO, L.; HAN, A.; ZIEGLER, S. F.; MATHIS, D.; BENOIST, C.; CHEN, L.; RAO, A. FOXP3 controls regulatory T cell function through cooperation with NF-AT. Cellular Immunology, New York, v. 126, p. 375-387, 2006.

ZHENG, Y.; JOSEFOWICZ, S. Z.; KAS, A.; CHU, T. T.; GAVIN, M. A.; RUDENSKY, A. Y. Genome-wide analysis of Foxp3 target genes in developing and mature regulatory T cells. Nature, London, v. 445, n. 7130, p. 936-940, 2007.

Recebido em: 15 de julho de 2009

Aceito em: 13 de agosto de 2010 